\title{
Thermodynamics of BTZ Black Holes in Gravity's Rainbow
}

\author{
Salwa Alsaleh \\ Department of Physics and Astronomy, King Saud University, Riyadh 11451, \\ Saudi Arabia \\ September 4, 2018
}

\begin{abstract}
In this paper, we deform the thermodynamics of a BTZ black hole from rainbow functions in gravity's rainbow. The rainbow functions will be motivated from results in loop quantum gravity and Noncommutative geometry. It will be observed that the thermodynamics gets deformed due to these rainbow functions, indicating the existence of a remnant. However, the Gibbs free energy does not get deformed due to these rainbow functions, and so the critical behaviour from Gibbs does not change by this deformation. This is because the deformation in the entropy cancel's out the temperature deformation.
\end{abstract}

\section{Introduction}

The Hořava-Lifshitz gravity is motivated by a deformation of the usual energy-momentum dispersion relation in the UV limit [35, 36. Another UV modification of general relativity also motivated by a deformation of the usual energy-momentum dispersion relation in the UV limit is the gravity's rainbow [41. It is interesting to note that the deformation of the usual energymomentum dispersion in the UV limit occurs in various approaches to quantum gravity such as discrete spacetime [48, models based on string field theory [38, spacetime foam [10, spin-network in loop quantum gravity (LQG) 23] and non-commutative geometry [19. The formalism has been used to study various geometries motivated from string theory. In fact, the different Lifshitz scaling of space and time has been used to deform type IIA string theory [28, type IIB string theory [17, AdS/CFT correspondence [29, 45, 7, 21, dilaton black branes [27, 15], cylindrical solutions 44 and dilaton black holes [52, 49] and. Gravity's rainbow is a more general theory that is motivated by deformation of the energy-momentum dispersion relation, similar to what motivates Hořava-Lifshitz gravity. Hence both approaches are connected to same quantum gravity phenomenology. In fact, for a particular choice of rainbow functions, gravity's rainbow seem's to agree with Hořava-Lifshitz gravity. As what has been shown in [26]. The Lifshitz deformation of geometries has produced interesting results, and rainbow deformation has the same motivation, in this paper we will study the rainbow deformation of BTZ black holes.

In gravity's rainbow, the geometry depends on the energy of the probe, and thus probes of of different energy see the geometry differently. Thus, a single metric is replaced by a family of energy dependent metrics forming a rainbow of metrics. Now the UV modification of the energy-momentum dispersion relation can be expressed as

$$
E^{2} f^{2}\left(E / E_{P}\right)-p^{2} g^{2}\left(E / E_{P}\right)=m^{2}
$$

where $E_{P}$ is the Planck energy, $E$ is the energy at which the geometry is probed, and $f\left(E / E_{P}\right)$ and $g\left(E / E_{P}\right)$ are the rainbow functions. As the general relativity should be recovered in the IR 
limit, we have

$$
\lim _{E / E_{P} \rightarrow 0} f\left(E / E_{P}\right)=1, \quad \lim _{E / E_{P} \rightarrow 0} g\left(E / E_{P}\right)=1 .
$$

Now the metric in gravity's rainbow 42

$$
h(E)=\eta^{a b} e_{a}(E) \otimes e_{b}(E) .
$$

So, the energy dependent frame fields are

$$
e_{0}(E)=\frac{1}{f\left(E / E_{P}\right)} \tilde{e}_{0}, \quad e_{i}(E)=\frac{1}{g\left(E / E_{P}\right)} \tilde{e}_{i} .
$$

Here $\tilde{e}_{0}$ and $\tilde{e}_{i}$ are the original energy independent frame fields.

The rainbow deformation of geometry motivated from string theory, such as black rings 2, and black branes [12] has been studied. The rainbow deformation of higher dimensional black holes has important consequences for the detection of black holes at the LHC 3 . The rainbow deformation of modified theories of gravity, and of gravity coupled to non-linear sources has been studied [30, 34, 33, 34, 34, 46, 32, 24]. The gravity's rainbow has also been used to address the information paradox in black holes [5] ?, 6]. It may be noted that general properties of energy dependent metric for a BTZ black hole, and its coupling to non-linear sources has been discussed using gravity rainbow 31. In this paper, we analyse the thermodynamic aspects of such a deformation explicitly. We are able to show that even though many thermodynamic quantities of a BTZ black hole are deformed by gravity's rainbow, the Gibbs free energy is not deformed. Thus, the critical phenomena based on Gibbs free energy is not deformed by gravity's rainbow.

\section{BTZ Black holes}

In $2+1$ dimensions, Einstein field equations with negative cosmological constant (AdS spacetime) admit - in addition to the vacuum solution- a two-parameter family of black hole solutions found by Banados, Teitelboim and Zanelli [14, given by the metric, without charge ${ }^{1}$

$$
d s^{2}=-N^{2} d t^{2}+N^{-2} d r^{2}+r^{2}\left(d \phi+N^{\phi} d t\right)^{2} .
$$

Where the functions $N^{2}=\frac{\left(r^{2}-r_{+}^{2}\right)\left(r^{2}-r_{-}^{2}\right)}{b^{2} r^{2}}$ and $\left(N^{\phi}\right)^{2}=\frac{r_{+} r_{-}}{b r^{2}}$. With $b$ is the radius of AdS and $r_{ \pm}$is obtained when the lapse function $N$ vanishes (indicating the outer and inner horizons, respectively),

$$
r_{ \pm}=\left[b^{2} \frac{M}{2}\left(1 \pm \sqrt{1-\frac{J^{2}}{b^{2} M^{2}}}\right)\right]^{1 / 2},
$$

with $M$ and $J$ being the mass and angular momentum of the BTZ black hole respectively. They can be therefore defined in terms of $b, r \pm$ accordingly:

$$
M=\frac{r_{+}^{2} r_{-}^{2}}{b^{2}}, \quad J=\frac{2 r_{+} r_{-}}{b} .
$$

In order to study the thermodynamics of BTZ black hole we first write the first law of black hole mechanics [47]

$$
d M=T d S+J d \Omega
$$

\footnotetext{
${ }^{1}$ Planckian units is used throughout the manuscript $k_{b}=c=G=\hbar=1$
} 
The angular speed $\Omega$ is calculated from $g_{t t} / g_{\phi \phi}$

$$
\Omega=\frac{J}{b^{2} M} .
$$

The temperature $T_{0}$ is calculating for the surface curvature, with the killing vector $K=\partial_{t}+\Omega \partial_{\phi}$, [16, 20]

$$
T_{0}=\frac{r_{+}^{2}-r_{-}^{2}}{2 \pi r_{+}} .
$$

The expression 10 indicates an extremal limit when $M<\underline{\text { J }}$. We observe that the temperature of

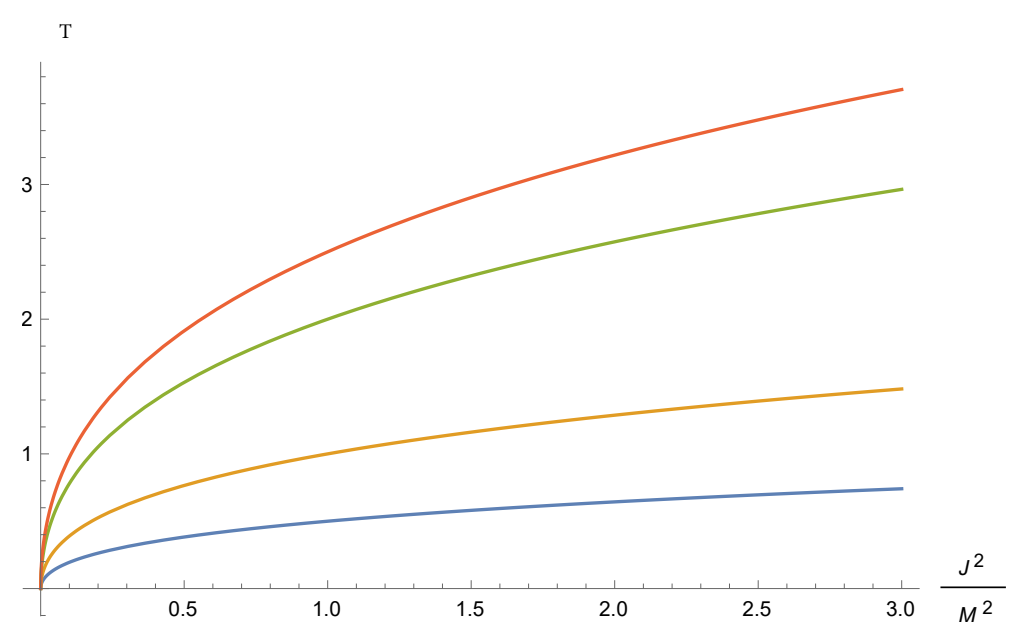

Figure 1: The temperature of different non-extremal BTZ black holes as a function of $\frac{J^{2}}{M^{2}}$. For different values of $b$. Blue: $b=0.25$,yellow: $b=0.5$, green: $b=0.75$ and red: $b=1$

BTZ black holes show a similar thermodynamic behaviour to their higher dimensional analogues figure 1. Now we use (8) and (7) to calculate the entropy

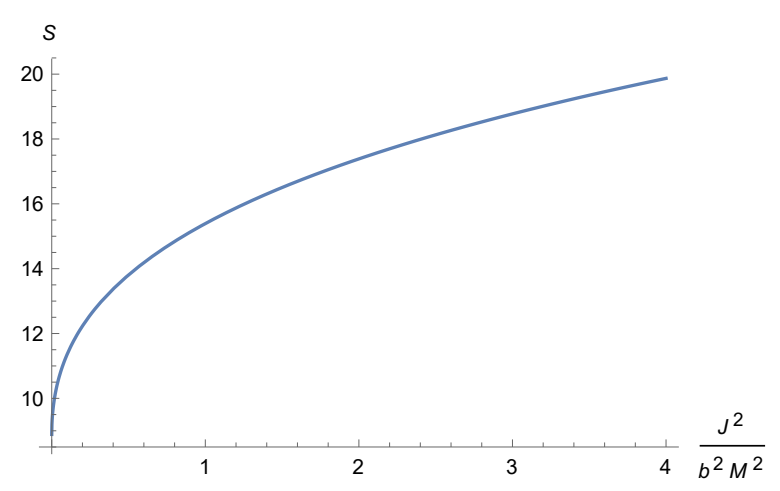

Figure 2: The Entropy of non-extremal BTZ black holes as a function of $\frac{J^{2}}{b^{2} M^{2}}$. Showing typical thermodynamic behaviour of Kerr-AdS black hole entropy

$$
S_{0}=4 \pi r_{+},
$$



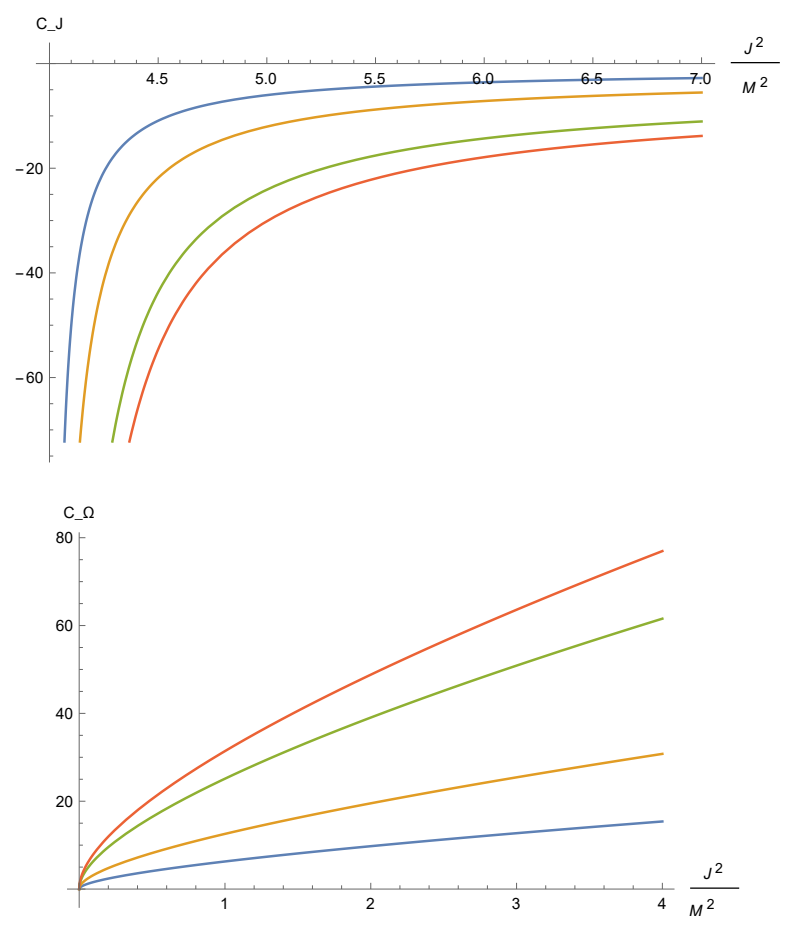

Figure 3: Figures show plots of $C_{J}$ and $C_{\Omega}$ for different BTZ black holes, as a function of $J^{2} /\left(M^{2}\right)$. For different values of $b$. Blue: $b=0.25$,yellow: $b=0.5$, green: $b=0.75$ and red: $b=1$

which is, as expected, the forth of the horizon area 'circumference'. Complying with the Bekenstein formula. We can also calculate the constant $J$ heat capacity $C_{J}$ :

$$
C_{J}=T\left(\frac{\partial S}{\partial T}\right)_{J}=\frac{4 \pi r_{+}}{2-\sqrt{1-\left(\frac{J}{b M}\right)^{2}}}\left[\frac{1+\sqrt{1-\left(\frac{J}{b M}\right)^{2}}\left(\frac{J}{b M}\right)^{2}}{2}\right]^{1 / 2}
$$

Similarly, the heat capacity at constant angular velocity $C_{\Omega}$ is calculated,

$$
C_{\Omega}=4 \pi b\left[\frac{M}{2}\left(1+\sqrt{1-\left(\frac{J}{b M}\right)^{2}}\right)\right]^{1 / 2} .
$$

We wish also to investigate the thermodynamic pressure - volume relation for BTZ black holes and the associated critical phenomena. We define the 'volume' of BTZ black hole by the relation [8, which is approximately the thermodynamic volume for slow rotating black holes $J \ll 1$.

$$
V_{0}=A r_{+}=16 \pi r_{+}^{2}
$$

Now, we consider the thermodynamic pressure of BTZ black hole from the Van der Wall's fluid equation of state in the extended phase space [39].

$$
P_{0}:=\frac{T}{v}+\mathcal{O}\left(J^{2}\right),
$$


with :

$$
v=2\left(\frac{V}{\pi}\right)^{1 / 2} .
$$

We observe from the PV diagram that BTZ black holes admit the same critical phenomena as the higher dimensional Kerr-AdS black holes for some critical temperature $T_{c}$ [22]. BTZ black

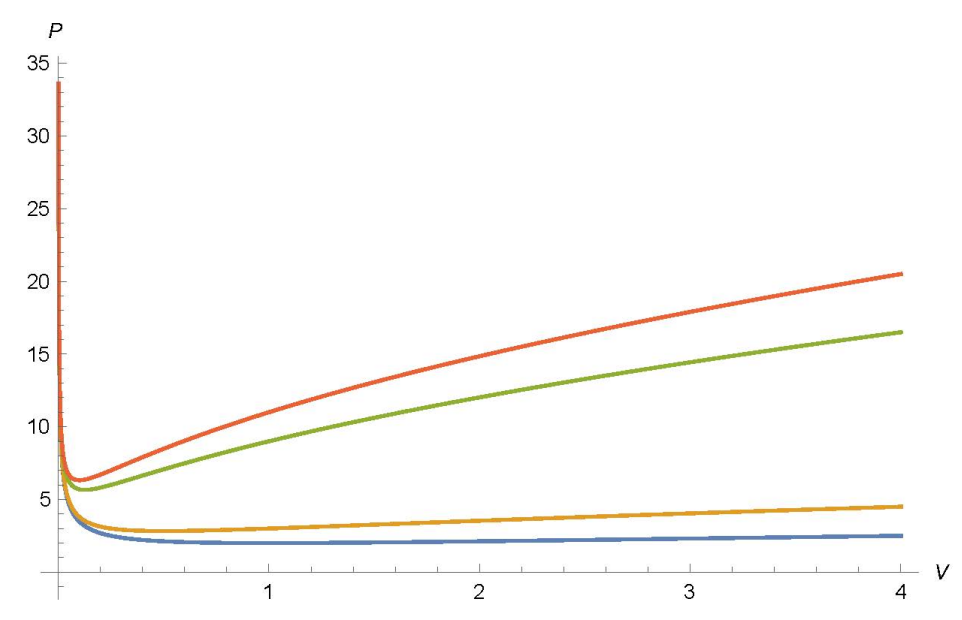

Figure 4: PV- diagram for different BTZ black holes, two showing a critical point $\partial P / \partial V=0$. For different values of $b$. Blue: $b=0.25$, yellow: $b=0.5$, green: $b=0.75$ and red: $b=1$

holes show an interesting thermodynamic properties. In the next section, they shall be studied after gravity rainbow deformation on the BTZ metric.

\section{BTZ black holes in gravity's rainbow}

In this section, we will deform the thermodynamics of a BTZ black hole by the gravity's rainbow. Now here $E$ is the energy of a quantum particle near the event horizon of the BTZ black hole. This particle is emitted from the black hole due to the Hawking radiation, the energy of the particle is associated with the black hole temperature $T$ [1]. In fact, in the geometric units used in the paper $k_{B}=1$, the black hole temperature is the same as the energy of the radiated particle, i.e $T_{B H}=E$. We can use the uncertainty principle, and write $\Delta p \geq 1 / \Delta x$. Thus, we can obtain a bound on energy of a black hole, $E \geq 1 / \Delta x[4$. This can be done for any black hole, including a BTZ black hole. It may be noted that the usual uncertainty principle is valid in gravity's rainbow [1]. So, the uncertainty in position of the particle near the horizon of the BTZ black hole is equal to the of the event horizon,

$$
E \geq 1 / \Delta x \approx 1 / r_{+} .
$$

It is important to noted from this that the energy used to deform the thermodynamics is a dynamical function of the radial coordinate [26] The general relation for temperature of a black hole in gravity rainbow was found to be [4] :

$$
T=T_{0} \frac{g(E)}{f(E)}
$$


Where $f(E)$ and $g(E)$ are the rainbow function defined in (2). This conjecture is explained and proved in the following references [6, 4, 11, 1, and many others. We may also show that the formula (18) applies for BTZ black holes in gravity rainbow as well.

First, consider the modification of the metric (5) by the gravity rainbow functions [31, 40, 6]:

$$
d s^{2}=-\frac{N^{2}}{f^{2}(E)} d t^{2}+g^{-2}(E) N^{-2} d r^{2}+g^{-2}(E) r^{2}\left(d \phi+N^{\phi} d t\right)^{2} .
$$

An the modified temperature is given by the generic formula [51]:

$$
T=\frac{1}{4 \pi} \sqrt{\partial_{r}(A(r, 0)) B(r, 0)}
$$

Where $A(r)=\frac{N^{2}}{f^{2}(E)}$ and $B(r)=g^{-2}(E) N^{-2}$. Therefore, we arrive at the formula for temperature in gravity rainbow 18 .

The are many ways in witch we can define the rainbow functions $f(E), g(E)$. Motivated by many theoretical [25, 13, 43]. And experimental [10] approaches. The choice of these functions in this paper is the one motivated by loop quantum gravity and non-commutative geometry [9, 37.

$$
f(E):=1 \quad g(E):=\sqrt{1-\eta\left(E / E_{p}\right)^{\nu}},
$$

for $\eta$ and $\nu$ being free parameters. Now, we use $(18)(10)$, and $(21)$ to obtain the modified BTZ temperature :

$$
T=\frac{r_{+}^{2}-r_{-}^{2}}{2 \pi r_{+}} \sqrt{1-\eta\left(1 / r_{+} E_{p}\right)^{\nu}}
$$

In order to calculate the modified entropy, we use the first law $d M=T d S$. With (7), and (11)

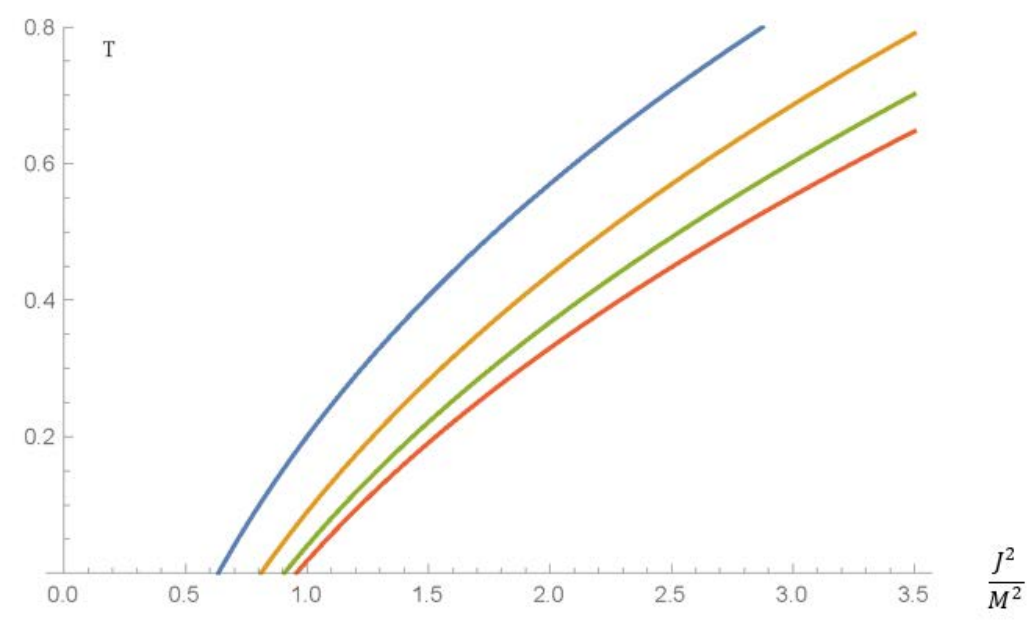

Figure 5: A plot of modified BTZ temperature as a function of $J^{2} / M^{2}$. For $E_{p}=5, \nu=1$ and $\eta=1$ for different values of $b$.Blue: $b=0.25$, yellow: $b=0.5$, green: $b=0.75$ and red: $b=1$. Showing the existence of a remnant point (vanishing temperature)

we get :

$$
S=\frac{\pi}{2} \frac{r_{+}}{g(E)}=\frac{\pi r_{+}}{2 \sqrt{1-\eta\left(1 / r_{+} E_{p}\right)^{\nu}}}
$$


It is interesting to look at the graphs between $S_{0}$ and $r_{+}$, and between $S$ and $r_{+} 6$. Observing that the entropy of rainbow gravity modified BTZ black holes will diminish at some point with $r_{+} \neq 0$, indicating the existence of remnant. This effect is observed in higher dimensional Kerr-AdS black holes in rainbow gravity [4. Since the entropy of a black hole is related to its

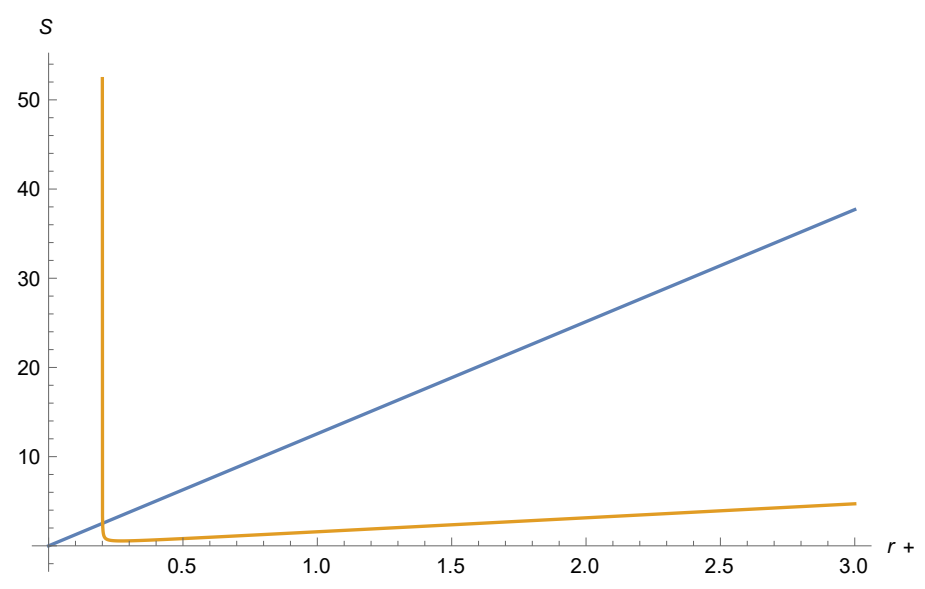

Figure 6: A plot of BTZ entropy $S_{0}$ in blue. And the deformed entropy $S$ in yellow (For $E_{p}=5$, $\eta=1$ and $\nu=1$ ), both as a function of $r_{+}$. The latter indicates the existence of remnant of radius $\frac{1}{5}$ Planck units.

area ( circumference in $2+1$ dimensions). Moreover, the $2+1$ dimensional gravity is a topological theory, one might relate the remnant of rainbow BTZ black hole as a topological defect associated with the minimal length of the $2+1$ gravity theory [50, 18. The radius of this topological defect is given by:

$$
r_{\text {min }}=\frac{\eta^{1 / \nu}}{E_{p}} \sim \ell_{p}
$$

Which could be the minimal length of the $2+1$ gravity rainbow. Now we calculate the modified heat capacities, observe that $\left(\frac{\partial S}{\partial T}\right)_{J}$ and $\left(\frac{\partial S}{\partial T}\right)_{\Omega}$, do not change by the gravity rainbow modifications. Hence the heat capacities are easily computed

$$
\begin{aligned}
& C_{J}=\sqrt{1-\eta\left(1 / r_{+} E_{p}\right)^{\nu}}\left[\frac{4 \pi r_{+}}{2-\sqrt{1-\left(\frac{J}{b M}\right)^{2}}}\right]\left[\frac{1+\sqrt{1-\left(\frac{J}{b M}\right)^{2}}\left(\frac{J}{b M}\right)^{2}}{2}\right]^{1 / 2} \\
& C_{\Omega}=4 \pi b \sqrt{1-\eta\left(1 / r_{+} E_{p}\right)^{\nu}}\left[\frac{M}{2}\left(1+\sqrt{1-\left(\frac{J}{b M}\right)^{2}}\right)\right]^{1 / 2} .
\end{aligned}
$$

The volume of the modified BTZ is given from (14):

$$
V=\frac{2 \pi r_{+}^{2}}{\sqrt{1-\eta\left(1 / r_{+} E_{p}\right)^{\nu}}}
$$

Moreover, the modified pressure is written as:

$$
P=\frac{1}{2}\left(1-\eta\left(1 / r_{+} E_{p}\right)^{\nu}\right)^{1 / 4} P_{0}
$$


The P-V criticality of rainbow BTZ is not different from the ordinary BTZ black hole. This also can be seen from calculating the Gibbs free energy of ordinary and rainbow BTZ black hole, The Gibbs free energy, $G$ given by the thermodynamic relation:

$$
G(T)=M+P V-T S .
$$

For an ordinary BTZ black holes it can be calculated using (7) (11) and (9).

$$
\begin{aligned}
G_{0}(T, \Omega)= & M-T S-\Omega J=-\frac{\pi^{2} b^{2}}{6 \sqrt{1-\left(\frac{J}{b M}\right)^{2}}} \\
& \left.\times \quad\left(T^{2}+\sqrt{\frac{2 \pi}{b}\left(1-\left(\frac{J}{b M}\right)^{2}\right.}\right) T+\frac{1}{4 \pi^{2} b^{2}}\left[2+\left(\frac{J}{b M}\right)^{2}\right]\right)\left(\frac{1}{b \Omega}\right)
\end{aligned}
$$

We may conclude that $G>0$ for critical BTZ black holes and $G<0$ for non critical ones

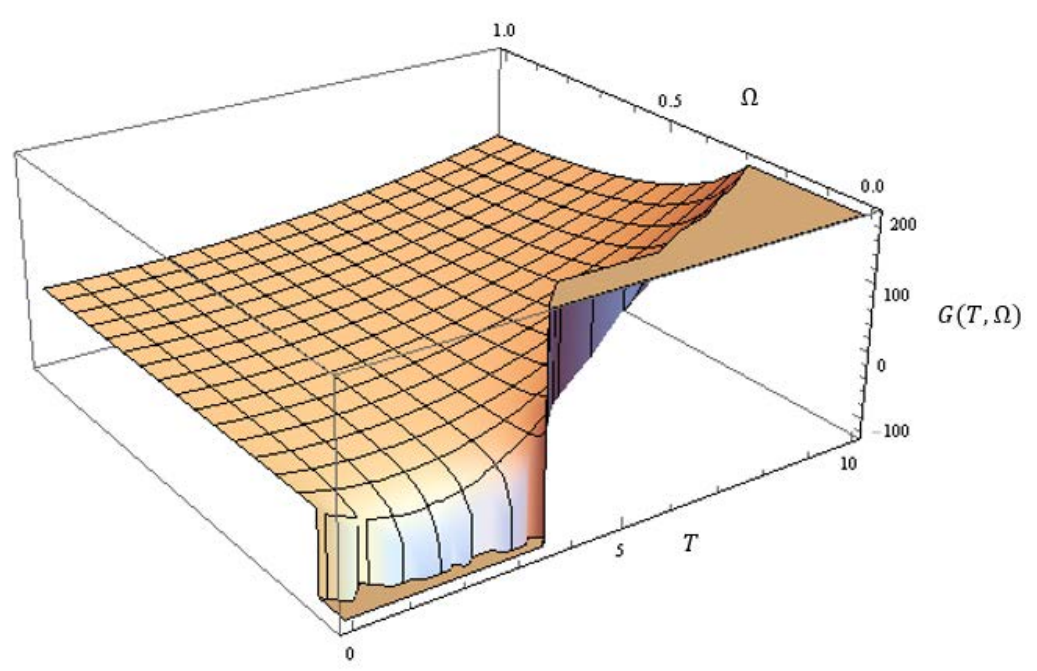

Figure 7: A plot of $G(T, \Omega)$ of a BTZ black hole $T>T_{c}$ with $b=0.5$. Showing the critical phenomena.

The Gibbs free energy for a rainbow BTZ is calculating using the same relation but, substituting $T_{0}$ and $S_{0}$ with $T$ in $(18)$ and $S$ in $(23)$. Since the rainbow function $g(E)$ appear in $T$ and its reciprocal appears in $S$. $G$ for the rainbow BTZ black hole is the same as $G_{0}$. Indicating the same critical phenomena.

\section{Conclusion}

In this paper, we have deformed the geometry of a BTZ black hole by rainbow functions. Thus, the thermodynamics of the BTZ black was also deformed by these rainbow functions. The rainbow functions that were used for this deformation have been motivated from results in loop quantum gravity and Noncommutative geometry. It was observed that the thermodynamics of the BTZ black hole got deformed due to these rainbow functions. The graphs of the deformed 
entropy $S$ and temperature $T$ indicate the existence of a remnant at the last stage of evaporation, similar to the higher dimensional deformed black holes. However, the Gibbs free energy did not get deformed, and so the critical behaviour from Gibbs did not change by this deformation. Thus, the critical behaviour of BTZ black holes in gravity's rainbow was the same the critical behaviour of BTZ black hole in ordinary gravity. This is apparent because the temperature is deformed in an opposite way to the entropy, causing both deformations to cancel out in the Gibb's free energy.

\section{Acknowledgements}

Warm regards to Dr Mir Faizal for his generous help improving this work.

This research project was supported by a grant from the " Research Center of the Female Scientiffic and Medical Colleges", Deanship of Scientiffic Research, King Saud University.

The author would like to thank the refrees for their helpful comments that improved the paper.

\section{References}

[1] Ahmed Farag Ali. Black hole remnant from gravitys rainbow. Physical Review D, 89(10):104040, 2014.

[2] Ahmed Farag Ali, Mir Faizal, and Mohammed M. Khalil. Remnants of black rings from gravity's rainbow. JHEP, 12:159, 2014.

[3] Ahmed Farag Ali, Mir Faizal, and Mohammed M. Khalil. Absence of Black Holes at LHC due to Gravity's Rainbow. Phys. Lett., B743:295-300, 2015.

[4] Ahmed Farag Ali, Mir Faizal, and Mohammed M Khalil. Remnant for all black objects due to gravity's rainbow. Nuclear Physics B, 894:341-360, 2015.

[5] Ahmed Farag Ali, Mir Faizal, and Barun Majumder. Absence of an Effective Horizon for Black Holes in Gravity's Rainbow. Europhys. Lett., 109(2):20001, 2015.

[6] Ahmed Farag Ali, Mir Faizal, Barun Majumder, and Ravi Mistry. Gravitational collapse in gravity's rainbow. International Journal of Geometric Methods in Modern Physics, 12(09):1550085, 2015.

[7] Mohsen Alishahiha and Hossein Yavartanoo. Conformally Lifshitz solutions from HoravaLifshitz Gravity. Class. Quant. Grav., 31:095008, 2014.

[8] Natacha Altamirano, David Kubizňák, Robert B Mann, and Zeinab Sherkatghanad. Thermodynamics of rotating black holes and black rings: phase transitions and thermodynamic volume. Galaxies, 2(1):89-159, 2014.

[9] Giovanni Amelino-Camelia. Quantum-spacetime phenomenology. Living Reviews in Relativity, 16(1):5, 2013.

[10] Giovanni Amelino-Camelia, John Ellis, NE Mavromatos, Dimitri V Nanopoulos, and Subir Sarkar. Tests of quantum gravity from observations of $\gamma$-ray bursts. Nature, 393(6687):763$765,1998$. 
[11] Marco Angheben, Mario Nadalini, Luciano Vanzo, and Sergio Zerbini. Hawking radiation as tunneling for extremal and rotating black holes. Journal of High Energy Physics, 2005(05):014, 2005.

[12] Amani Ashour, Mir Faizal, Ahmed Farag Ali, and Fayçal Hammad. Branes in gravitys rainbow. The European Physical Journal C, 76(5):1-9, 2016.

[13] Adel Awad, Ahmed Farag Ali, and Barun Majumder. Nonsingular rainbow universes. Journal of Cosmology and Astroparticle Physics, 2013(10):052, 2013.

[14] Maximo Banados, Claudio Teitelboim, and Jorge Zanelli. Black hole in three-dimensional spacetime. Physical Review Letters, 69(13):1849, 1992.

[15] Gaetano Bertoldi, Benjamin A Burrington, and Amanda W Peet. Thermodynamics of black branes in asymptotically lifshitz spacetimes. Physical Review D, 80(12):126004, 2009.

[16] Danny Birmingham, Ivo Sachs, and Siddhartha Sen. Exact results for the btz black hole. International Journal of Modern Physics D, 10(06):833-857, 2001.

[17] Philipp Burda, Ruth Gregory, and Simon Ross. Lifshitz flows in IIB and dual field theories. JHEP, 11:073, 2014.

[18] Steven Carlip. The $(2+1)$-dimensional black hole. Classical and Quantum Gravity, 12(12):2853, 1995.

[19] Sean M Carroll, Jeffrey A Harvey, V Alan Kosteleckỳ, Charles D Lane, and Takemi Okamoto. Noncommutative field theory and lorentz violation. Physical Review Letters, 87(14):141601, 2001.

[20] Norman Cruz and Samuel Lepe. On the thermal description of the btz black holes. Physics Letters B, 593(1):235-241, 2004.

[21] Parijat Dey and Shibaji Roy. Interpolating solution from ads 5 to hyperscaling violating lifshitz spacetime. Physical Review D, 91(2):026005, 2015.

[22] Antonia M. Frassino, David Kubiznak, Robert B. Mann, and Fil Simovic. Multiple Reentrant Phase Transitions and Triple Points in Lovelock Thermodynamics. JHEP, 09:080, 2014.

[23] Rodolfo Gambini and Jorge Pullin. Nonstandard optics from quantum space-time. Phys. Rev., D59:124021, 1999.

[24] Remo Garattini. Distorting General Relativity: Gravity's Rainbow and $\mathrm{f}(\mathrm{R})$ theories at work. JCAP, 1306:017, 2013.

[25] Remo Garattini and Barun Majumder. Electric charges and magnetic monopoles in gravity's rainbow. Nuclear Physics B, 883:598-614, 2014.

[26] Remo Garattini and Emmanuel N. Saridakis. Gravitys Rainbow: a bridge towards HoavaLifshitz gravity. Eur. Phys. J., C75(7):343, 2015.

[27] Kevin Goldstein, Norihiro Iizuka, Shamit Kachru, Shiroman Prakash, Sandip P. Trivedi, and Alexander Westphal. Holography of Dyonic Dilaton Black Branes. JHEP, 10:027, 2010.

[28] Ruth Gregory, Susha L Parameswaran, Gianmassimo Tasinato, and Ivonne Zavala. Lifshitz solutions in supergravity and string theory. Journal of High Energy Physics, 2010(12):1-18, 2010 . 
[29] Steven S. Gubser and Abhinav Nellore. Ground states of holographic superconductors. Phys. Rev., D80:105007, 2009.

[30] S. H. Hendi, S. Panahiyan, B. Eslam Panah, and M. Momennia. Thermodynamic instability of nonlinearly charged black holes in gravitys rainbow. Eur. Phys. J., C76(3):150, 2016.

[31] Seyed Hossein Hendi. Asymptotically charged btz black holes in gravitys rainbow. General Relativity and Gravitation, 48(4):1-15, 2016.

[32] Seyed Hossein Hendi, Ali Dehghani, and Mir Faizal. Black hole thermodynamics in Lovelock gravity's rainbow with (A)dS asymptote. Nucl. Phys., B914:117-137, 2017.

[33] Seyed Hossein Hendi and Mir Faizal. Black holes in Gauss-Bonnet gravitys rainbow. Phys. Rev., D92(4):044027, 2015.

[34] SH Hendi, S Panahiyan, S Upadhyay, and B Eslam Panah. Charged btz black holes in the context of massive gravity's rainbow. arXiv preprint arXiv:1611.02937, 2016.

[35] Petr Hořava. Quantum gravity at a lifshitz point. Physical Review D, 79(8):084008, 2009.

[36] Petr Hořava. Spectral dimension of the universe in quantum gravity at a lifshitz point. Physical review letters, 102(16):161301, 2009.

[37] Uri Jacob, Flavio Mercati, Giovanni Amelino-Camelia, and Tsvi Piran. Modifications to lorentz invariant dispersion in relatively boosted frames. Physical Review D, 82(8):084021, 2010.

[38] V. Alan Kostelecky and Stuart Samuel. Spontaneous Breaking of Lorentz Symmetry in String Theory. Phys. Rev., D39:683, 1989.

[39] David Kubizñák and Robert B Mann. P- v criticality of charged ads black holes. Journal of High Energy Physics, 2012(7):1-25, 2012.

[40] Zheng Ze Ma. Hawking temperature of kerr-newman-ads black hole from tunneling. Physics Letters B, 666(4):376-381, 2008.

[41] Joao Magueijo and Lee Smolin. Lorentz invariance with an invariant energy scale. Physical Review Letters, 88(19):190403, 2002.

[42] Joao Magueijo and Lee Smolin. Gravity's rainbow. Classical and Quantum Gravity, 21(7):1725, 2004.

[43] Barun Majumder. Singularity free rainbow universe. International Journal of Modern Physics D, 22(12):1342021, 2013.

[44] Davood Momeni, Sudhaker Upadhyay, Yerlan Myrzakulov, and Ratbay Myrzakulov. Cosmic string in gravity's rainbow. 2017.

[45] Yen Chin Ong and Pisin Chen. Stability of Horava-Lifshitz Black Holes in the Context of AdS/CFT. Phys. Rev., D84:104044, 2011.

[46] Prabir Rudra, Mir Faizal, and Ahmed Farag Ali. Vaidya Spacetime for Galileon Gravity's Rainbow. Nucl. Phys., B909:725-736, 2016.

[47] Larry Smarr. Mass formula for kerr black holes. Physical Review Letters, 30(2):71, 1973. 
[48] Gerard 't Hooft. Quantization of point particles in (2+1)-dimensional gravity and space-time discreteness. Class. Quant. Grav., 13:1023-1040, 1996.

[49] Javier Tarrio and Stefan Vandoren. Black holes and black branes in Lifshitz spacetimes. JHEP, 09:017, 2011.

[50] Alexander Vilenkin and E Paul S Shellard. Cosmic strings and other topological defects. Cambridge University Press, 2000.

[51] Robert M Wald. General relativity. University of Chicago press, 2010.

[52] M. Kord Zangeneh, A. Sheykhi, and M. H. Dehghani. Thermodynamics of topological nonlinear charged Lifshitz black holes. Phys. Rev., D92(2):024050, 2015. 